\title{
Improving Vision-Based Motor Rehabilitation Interactive Systems for Users with Disabilities Using Mirror Feedback
}

\author{
Antoni Jaume-i-Capó, ${ }^{1}$ Pau Martínez-Bueso, ${ }^{2}$ Biel Moyà-Alcover, ${ }^{1}$ and Javier Varona ${ }^{1}$ \\ ${ }^{1}$ Unitat de Gràfics, Visió i Intelligència Artificial, Department de Ciències Matemàtiques i Informàtica, Universitat de les Illes Balears, \\ E07122 Palma, Spain \\ ${ }^{2}$ Grup d'Investigació d'Evidència, Estils de Vida i Salut, Department d'Infermeria i Fisioteràpia, Universitat de les Illes Balears, \\ E07122 Palma, Spain
}

Correspondence should be addressed to Antoni Jaume-i-Capó; antoni.jaume@uib.es

Received 17 May 2014; Revised 5 August 2014; Accepted 22 August 2014; Published 11 September 2014

Academic Editor: Ching-Yi Wu

Copyright (C) 2014 Antoni Jaume-i-Capó et al. This is an open access article distributed under the Creative Commons Attribution License, which permits unrestricted use, distribution, and reproduction in any medium, provided the original work is properly cited.

\begin{abstract}
Observation is recommended in motor rehabilitation. For this reason, the aim of this study was to experimentally test the feasibility and benefit of including mirror feedback in vision-based rehabilitation systems: we projected the user on the screen. We conducted a user study by using a previously evaluated system that improved the balance and postural control of adults with cerebral palsy. We used a within-subjects design with the two defined feedback conditions (mirror and no-mirror) with two different groups of users (8 with disabilities and 32 without disabilities) using usability measures (time-to-start $\left(T_{s}\right)$ and time-to-complete $\left(T_{c}\right)$ ). A two-tailed paired samples $t$-test confirmed that in case of disabilities the mirror feedback facilitated the interaction in vision-based systems for rehabilitation. The measured times were significantly worse in the absence of the user's own visual feedback $\left(T_{s}=7.09(P<0.001)\right.$ and $\left.T_{c}=4.48(P<0.005)\right)$. In vision-based interaction systems, the input device is the user's own body; therefore, it makes sense that feedback should be related to the body of the user. In case of disabilities the mirror feedback mechanisms facilitated the interaction in vision-based systems for rehabilitation. Results recommends developers and researchers use this improvement in vision-based motor rehabilitation interactive systems.
\end{abstract}

\section{Introduction}

Video game console technologies focusing on motion-based inputs, designed to track body motions or body reactions (e.g., EyeToy, Wiimote, Kinect, Xtion, and Move), are becoming popular and low-cost $[1,2]$. These sensors can capture motions of the motor therapy and different studies validated Kinect sensor for rehabilitation purposes such as postural control [3], clinical functional analysis and rehabilitation [4], gait retraining [5], activities of daily living rehabilitation [6], and coaching of elderly population [7].

Recent research showed that video games helped to motivate patients in rehabilitation processes [8-10] and rehabilitation results were better with motivated patients [11]. However, different studies concluded that existing commercial motion-based video games were difficult to use in rehabilitation therapy, because they were designed for users with full capabilities $[12,13]$. Therefore, researchers developed motion-based video games for motor rehabilitation using the existing commercial motion-based devices: pressure mat based for maintenance of balance in a short-sitting position following spinal cord and head injuries [14]; vision-based for upper limb stroke rehabilitation [15], for chronic stroke recovery [16-18] and to improve the balance and postural control of adults with cerebral palsy [19]; wiimote-based for postural control and functional mobility of cerebral palsy patients [20]; Kinect-based to guide and correct therapeutic movements [21], to train static balance [22], and to improve the motor proficiency and quality of life [23]; and hapticbased for stroke rehabilitation [24]. Also, literature reviews about motion-based rehabilitation system were published in the last years $[8,9,25,26]$.

In particular, Kinect sensor captures the visual information of the performance of user motions; then it can 
also consider a vision-based interaction sensor (VBI) [27]. Visual information from the performance of patient actions is a good capture method in motor rehabilitation for two reasons: first, because motor rehabilitation consists of body movements that can be recorded; and second, because VBI is noninvasive and can be used for clients who have difficulties in holding physical devices.

In rehabilitation systems using VBI is critical to provide feedback to users in order to feel in control and help them to understand what is happening $[15,19,28]$. In VBI there is not contact with the interface by means of an interaction device of reference. The user, therefore, always should know when interaction is taking place using visual and audible feedback. For instance, in prior experiences developing vision-based video games for hospitals, medical institutions, and rehabilitation centres $[10,19,29-31]$, we detected that users had some difficulties in performing the therapy movements if they could not see themselves in a mirror or on screen.

The advantage of observation and imitation for learning is well studied $[32,33]$, and mirror movements and imitation learning is recommended in motor rehabilitation [34]. Motor control amends the motion by interaction between visual feedback that recognizes the external space or movement of oneself through vision feedback that refers information about movement and position of body [35]. Moreover, there is evidence that action observation facilitates motor activity [36]. For this reason, mirrors equip motor therapy rooms and they allow the patients to see themselves in order to perform correctly the therapy.

In fact, some exiting VBI rehabilitation systems allow the patient seeing themselves on screen, mirror feedback, due to the fact that the users stand in front of a screen and interact with the system using their movements $[15,16,23]$; see Figure 1. It was demonstrated that the user's own image suggested more realism and sense of presence than an avatar figure [37]. The more sense of presence the users have, the more aware of their position and orientation with respect to the interaction elements the users are. Nevertheless, other VBI rehabilitation systems do not implement the mirror feedback [17, 21, 22] because game-based rehabilitation systems designers frequently overemphasize the video game rather than the user interaction. When these games are designed for people with disabilities, the interaction design issues are fundamental to achieve a high patient's motivation. In addition, game interaction design is usually defined without taking into account user's perceptions with regard to their actions in order to achieve the rehabilitation goals.

Different researchers studied the importance and the effectiveness of the augmented feedback in the therapy (information that cannot be elaborated without an external source such as a therapist or a device) [38, 39]. They discovered that visual augmented feedback could improve the performance of the patients on complex motor tasks. However, to our knowledge, there did not exist any study about the importance of the mirror feedback in visionbased rehabilitation systems. Concretely, Sigrist's survey [39] reviewed different types of natural visualization feedback (such as superposition, side-by-side 3D perspective, endeffector movements, and third- and first-person perspective) and only introduced the mirrors in the case of mirror therapy [40].

Our objective was to explore how mirror feedback through interaction could be included into game interaction design in order to observe whether it was possible to improve results in rehabilitation sessions. We performed a user study testing using a game previously designed for balance rehabilitation of cerebral palsy users to explore mirror feedback in vision-based video games. Participants diagnosed with cerebral palsy (CP) with mild to moderate cognitive impairment performed our user study. CP is the most common cause of disabling conditions in children due to the increased survival of low birth-weight infants [41]. The population of adults with $\mathrm{CP}$ is growing, as a result of increased longevity, inspiring new research to improve available therapies to achieve better functional abilities. We chose adults with CP for this reason and because many daily activities require both hands and tasks that typically require bimanual coordination [42, 43]. The aim was to experimentally test that the feedback of current games was not enough for understanding the game play by users with cognitive impairment and to demonstrate the feasibility and benefit of including mirror feedback in VBI rehabilitation systems.

The remainder of this paper is organized as follows. The experimental system is presented in Section 2. In Section 3, the experiment is designed and in Section 4 we show the results. Finally, Section 5 discusses the issues observed and the last section is devoted to conclusions and proposed further work.

\section{Experimental System}

The experimental system had been designed to improve the balance and postural control of adults with CP [19]. The system was based on a video game for balance rehabilitation therapy, designed using the prototype development paradigm and features for rehabilitation with video games [15, 28, 44]: feedback, adaptability, motivational elements, and monitoring. We rigorously evaluated the effects of physiotherapy treatment on balance postural control of adult subjects with CP undergoing our experimental system. A 24-week physiotherapy intervention program was conducted with 9 adults from a CP center who exercised weekly in 20-minute sessions. Findings demonstrated a significant increase in balance postural control scores resulting in indicators of greater independence for our participating adults. Scores improved from 16 to 21 points on a scale of 28 , according to the Tinetti Scale for risk of falls, moving from High Fall Risk to Moderate Fall Risk.

We used an active control therapy [45] as interaction method for a video game to improve balance and postural control, increase motivation in clients, and achieve higher adherence to this long-term therapy. The users must interact with objects that cannot be reached without moving the center of mass beyond the base of support (see Figure 2). More specifically, users must remove individual items that appear on the screen by reaching each item with one hand.

The video game responds to the actions of the user through different types of feedback, in order for the users to be aware of their current state; see Figure 3. 


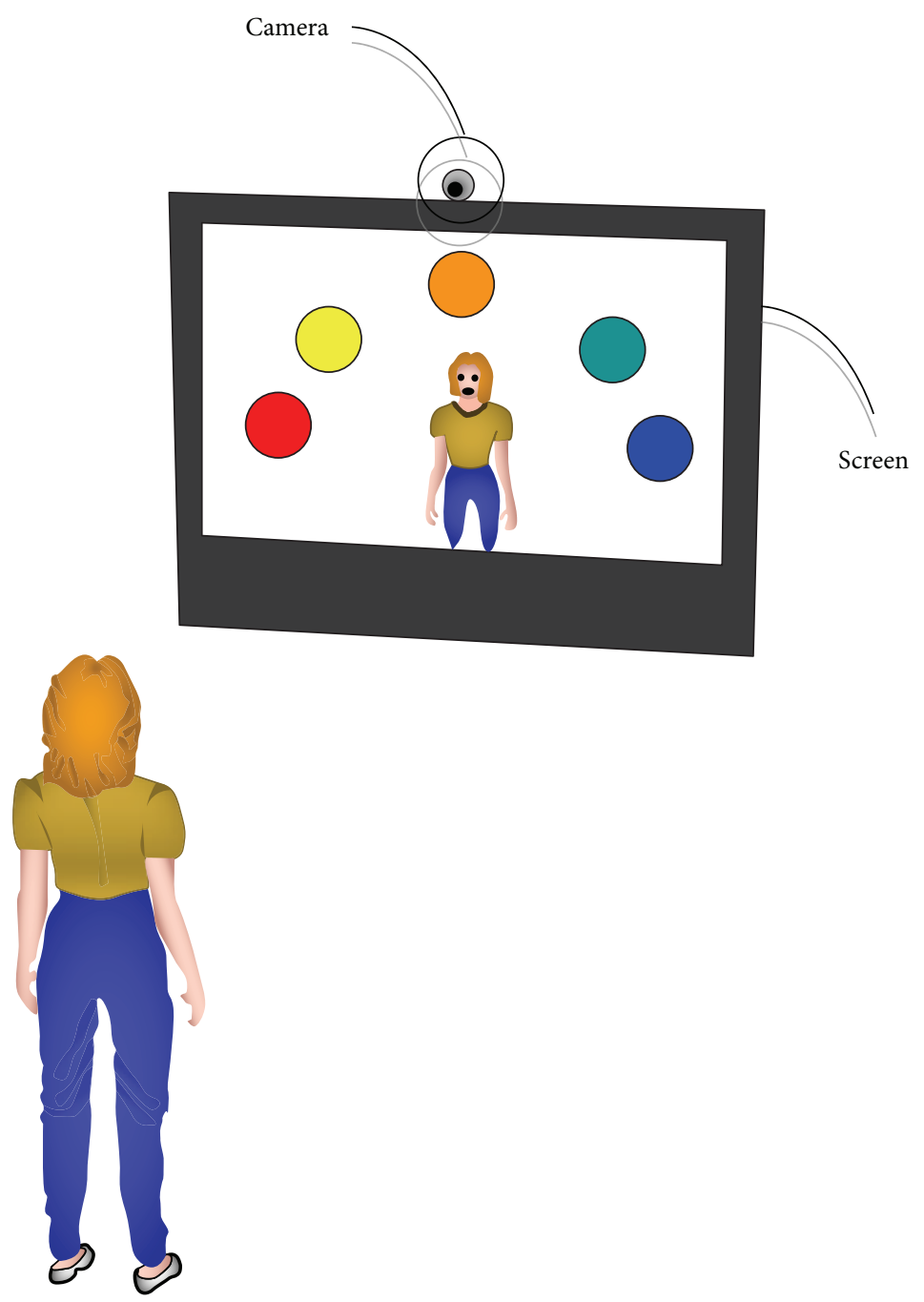

FIGURE 1: System environment configuration.

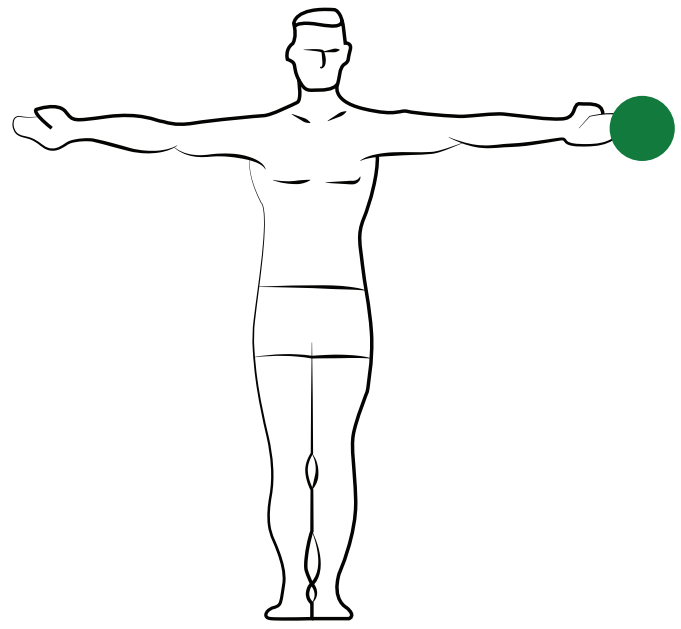

FIGURE 2: The user is within normal reach of the green object but must change the centre of mass to reach and grab the red object. (i) A pointer is projected on the user's hand, and the part of the interaction object that intersects with the pointer is erased.

(ii) An auditory feedback is played when an interaction object is completely deleted from the screen.

(iii) Mirror feedback is considered, in such a way that the user can see himself/herself on the screen at all times, so the player's position relative to interaction objects is always known.

Moreover, when the game ends, the user receives different types of visual and auditory feedback, depending on the end game conditions.

The experimental system was developed using $\mathrm{C}++$ programming language, OpenNI as Kinect device library, OpenCV as a computer vision library, and Qt as the graphical user interface library. 


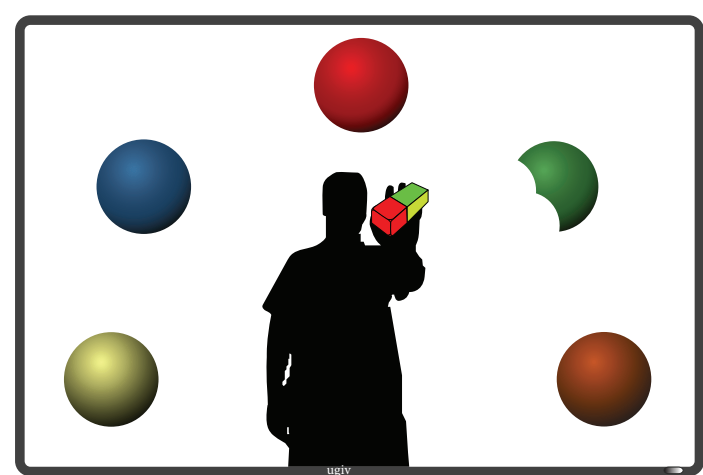

FIGURE 3: Rehabilitation session introducing mirror feedback.

\section{Experiment}

The experiment performed in this work was aimed at validating that mirror feedback mechanism was important for vision-based rehabilitation systems, especially for the characteristics of the users' set presented.

3.1. Participants. Adults diagnosed with $\mathrm{CP}$ and with limited voluntary motor control of one or both arms and legs and of the trunk were recruited from the Spanish Association of Cerebral Palsy Centre (ASPACE) in the Balearic Islands. These subjects had mild to moderate cognitive impairment, as shown in Table 1. We used the mini-mental state examination (MMSE) to classify their cognitive impairment because it is a brief and objective screening test and also because it is valid and reliable across a variety of clinical, epidemiological, and community survey studies [46]. The inclusion criteria were as follows:

(i) age of 20 to 65 years;

(ii) no participation in clinical study published in [19];

(iii) ability to walk with or without technical aids (GMFCS I and II) (Gross Motor Function Classification System);

(iv) ability to understand, learn, and follow simple instructions;

(v) voluntary agreement to participate in the clinical study.

The exclusion criteria were as follows:

(i) severe cognitive impairment;

(ii) profound bilateral hearing loss with the use of hearing aids;

(iii) severe visual impairment;

(iv) serious or uncontrolled epilepsy;

(v) serious or recurring medical complications.

The research team made a request to all adults in the ASPACE. The final study population included 8 adults (7 males), aged 22 to 41 (mean $(M)=33$ ), with CP. Their families signed an informed consent, as legal proxies. Characteristics of the participants are presented in Table 1.
TABLE 1: Characteristics of participants.

\begin{tabular}{lccc}
\hline User & Age & Physical diagnosis & MMSE \\
\hline 1 & 22 & Cerebral palsy & Moderate \\
& & spastic tetraparesis & Cognitive impairment \\
2 & 27 & Cerebral palsy & Mild \\
& & spastic tetraparesis & cognitive impairment \\
3 & 32 & Cerebral palsy & Moderate \\
& & spastic tetraparesis & cognitive impairment \\
4 & 32 & Cerebral palsy & Mild \\
& & mixed spastic tetraparesis & cognitive impairment \\
5 & 34 & Cerebral palsy & Mild \\
& & spastic tetraparesis & cognitive impairment \\
6 & 37 & Head trauma & Mild \\
& & spastic tetraparesis & cognitive impairment \\
7 & 39 & Cerebral palsy & Moderate \\
& & mixed spastic tetraparesis & cognitive impairment \\
8 & 41 & Cerebral palsy & Mild \\
& & ataxic tetraparesis & cognitive impairment \\
\hline
\end{tabular}

We also included a control group composed of 32 nonpaid volunteers (14 females) aged 19 to $25(\mathrm{M}=20.4)$, with no disabilities.

3.2. Procedure. In order to explore the importance of mirror feedback in vision-based motor rehabilitation interactive systems, we conducted a user study testing. Specifically, the users tested their own visual representation such as interaction feedback of the video game for rehabilitation.

We were interested in the different users performances in the game interaction enabling or not the explained mirror feedback mechanism; that is, the no-mirror feedback condition was characterized by the absence of such visual feedback. Other feedback mechanisms defined in Section 2 were activated.

We used a within-subjects design with the two previously defined feedback conditions:

MF: mirror feedback (including the user's own visual representation);

NM: no-mirror feedback (absence of such visual feedback).

In Figure 4 it is possible to observe the feedback for the two interaction conditions. The user study was divided into two experiments with two different groups of users:

C: control group (users without disabilities);

D: users with disabilities.

For the control group, the user study started with a brief introduction and a demonstration, together with a demographic questionnaire asking about age and previous use of vision-based interaction applications. Participants played two sessions of the designed computer game with the same conditions that the user with disabilities, that is, only moving the upper body part to delete the virtual objects with their hands. For each session the order of conditions (mirror feedback 


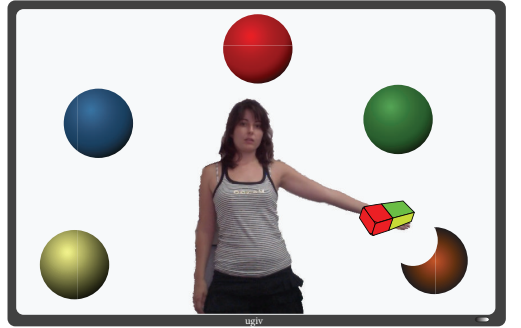

(a) Mirror feedback

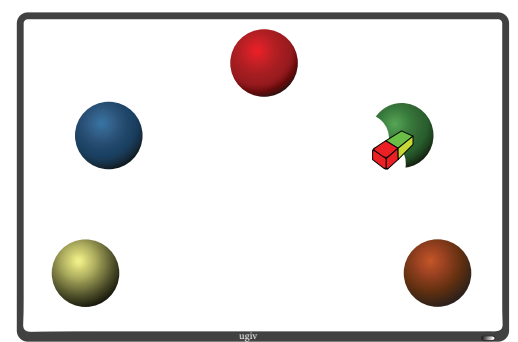

(b) No-mirror feedback

FIGURE 4: Experimental feedback conditions.

versus no-mirror feedback) was randomly selected so as to balance both interaction conditions across participants.

For users with disabilities, the game was previously tested on a pilot scheme for a two-month period, attending the rehabilitation center once a week. They practiced the game for at least 20 minutes only with no-mirror feedback condition, and the number of repetitions varied according to participants' tolerance and the physiotherapist's prescription to better manage fatigue. These two months of training were important to ensure a correct understanding of the game and to learn how to carry it out, as well as ensuring a correct parameter adaptation to each user. Once the users correctly understood the game play, participants played two sessions of the designed computer applying the same procedure as for the control group. Figure 5 shows real performance of the system in ASPACE rehabilitation room using mirror feedback.

3.3. Measurements. The gaming was assisted by the physiotherapist and monitored by the research team. All experiments were performed using a PC with this configuration:

(i) Intel Core2 Duo CPU P8400 @2,26 Ghz;

(ii) 3034 MB RAM;

(iii) Graphic card Mesa DRI mobile Intel GM45 Express;

(iv) Ubuntu 9.10;

(v) Microsoft Kinect.

With this configuration the system performance was $30 \mathrm{fps}$. This result ensured a real-time response from the system [47].

Quantitative measures included logged time-to-start $\left(T_{s}\right)$ and time-to-complete $\left(T_{c}\right)$ times. The time-to-start measured the time the user interacted with the first virtual object. We interpreted this time as the time taken by the users to orientate their motions with the game interactions. This measure was derived from the observations in the pretest sessions performed with the pilot. In these sessions, users with disabilities had greater difficulty in attaining orientation, and they had trouble knowing their position during play, relative to the interaction objects. This fact was more clearly observable when they had to delete the first virtual object.

The time-to-complete measured the time that users needed to complete the deletion of all virtual objects. In the experiment with the group of users with disabilities, the virtual objects were properly located in order to ensure that all
TABLE 2: Measured time-to-start $\left(T_{s}\right)$ and time-to-complete $\left(T_{c}\right)$ for users with disabilities. Mirror feedback (MF) and no-mirror feedback (NM).

\begin{tabular}{lcccc}
\hline User & $T_{s}$ & $T_{s}$ & $T_{c}$ & $T_{c}$ \\
& MF & NM & MF & NM \\
\hline 1 & 5 & 23 & 150 & 245 \\
2 & 2 & 11 & 129 & 160 \\
3 & 5 & 26 & 132 & 226 \\
4 & 2 & 10 & 126 & 154 \\
5 & 2 & 10 & 160 & 174 \\
6 & 3 & 15 & 132 & 176 \\
7 & 4 & 19 & 148 & 218 \\
8 & 2 & 11 & 121 & 148 \\
\hline
\end{tabular}

the performances achieved the complete deletion goal. Furthermore, in a final questionnaire, the participants selected their preferred interaction feedback for playing the game.

The time-to-start is related to effectiveness and time-tocomplete is related to efficiency of interaction task. According to usability definition [48], it has three aspects: satisfaction, effectiveness, and efficiency. Satisfaction's measures include users' preferences: we had demonstrated that experimental system improved the balance and postural control [19]; that is, the user's objective and the final questionnaire indicated they preferred interaction feedback for playing the game. Effectiveness's measures include quality of solution: time-tostart implies first interaction, and users are not able to complete the task if they do not understand the game mechanics and, therefore, start to play. Then, it also has a direct correlation with the task completion and the quality of solution. Efficiency's measures include use of time: tasks completion time (time-to-complete).

\section{Results}

Table 2 shows the measured time-to-start and time-tocomplete for users with disabilities using the feedback conditions defined by the experiment. Mirror feedback had better results on the measured times for users with disabilities. Users with moderate cognitive impairment had bigger differences between feedback conditions (mirror versus no-mirror) than users with mild cognitive impairment. 
TABLE 3: Overview of the influence of mirror feedback as interaction feedback for each user group on the mean of the defined time measures.

\begin{tabular}{lcr}
\hline & Time-to-start, $T_{s}$ & Time-to-complete, $T_{c}$ \\
\hline Control group $(\mathbf{C})$ & $t(31)=-1.74, P=0.09$ & $t(31)=-0.87, P=0.389$ \\
Users with disabilities (D) & $t(7)=7.09, P<\mathbf{0 . 0 0 1}$ & $t(7)=4.48, P<\mathbf{0 . 0 0 5}$ \\
\hline
\end{tabular}

Significant results are printed in bold.

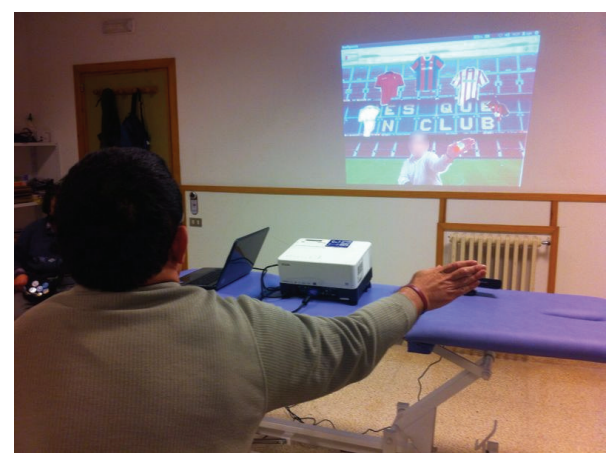

FIGURE 5: Real performance of the system in ASPACE rehabilitation room using mirror feedback.

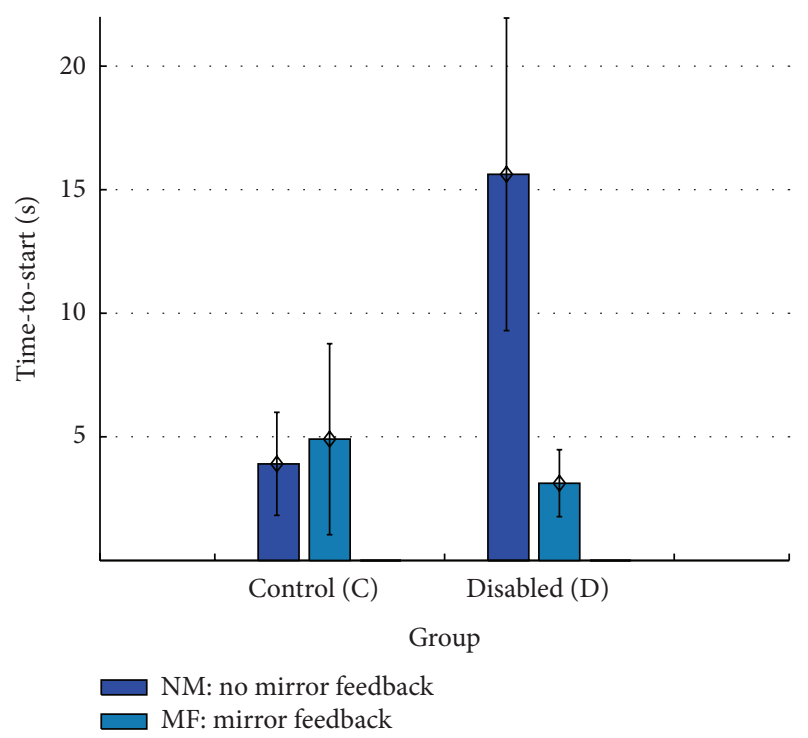

Figure 6: Overview of mean times for the time-to-start measure $\left(T_{s}\right)$.

Figures 6 and 7 show mean completed times for both measures, with error bars indicating $95 \%$ confidence intervals (CI). They will be further discussed in the following section, based on the interaction feedback. Table 3 summarizes the influence of mirror feedback in the mean measured times for $T_{s}$ and $T_{t}$.

A two-tailed paired samples $t$-test was conducted to evaluate the impact of mirror feedback for each measure in both user groups. Mirror feedback had a highly significant impact on the measured times for users with disabilities (cf. Table 3). The measured times were significantly worse in the absence of the user's own visual feedback. The control group, on the other hand, completed the experiment with both feedback

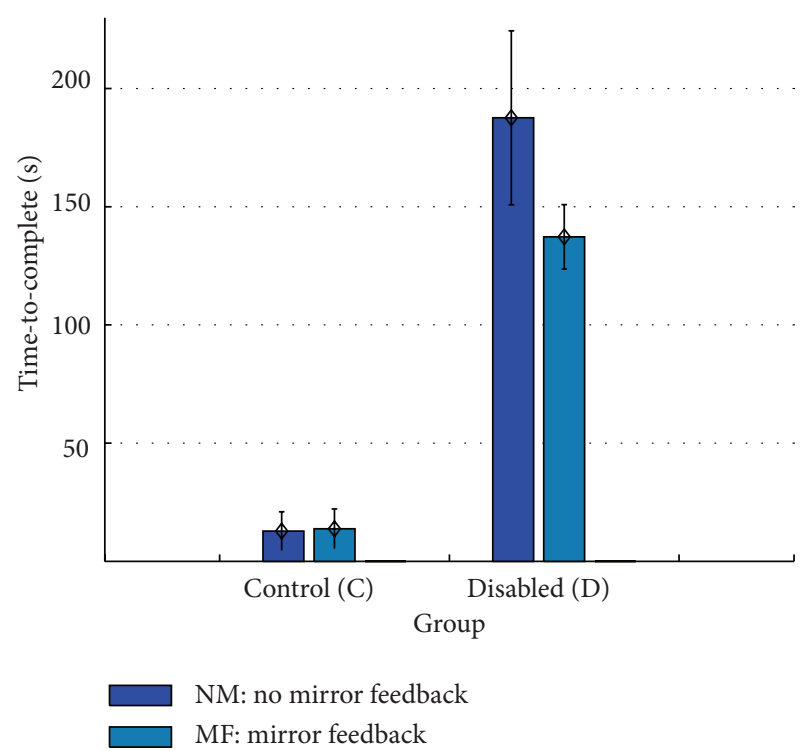

FIGURE 7: Overview of mean times for the time-to-complete measure $\left(T_{c}\right)$.

types with no significant performance differences. However, in the final questionnaire results about interaction feedback preferences, 24 participants of the control group preferred the mirror feedback. For the group with disabilities, seven participants answered that by including mirror feedback mechanisms they gained more control.

Before using the paired $t$-test, we applied a KolgomorovSmirnov test of normality $(D=0.2587, P=0.1187)$ and the Wilcoxon signed rank test with continuity correction obtaining a $P$ value less than $0.01(P=0.007015)$.

\section{Discussion}

Results confirmed our hypothesis that in case of disabilities the mirror feedback mechanisms facilitated the interaction in vision-based systems for rehabilitation. They demonstrated that the implementation of mirror feedback by giving patients the possibility of seeing themselves on screen means that they were conscious at all times of the actions performed relative to the video game. In the user study presented, we proved this claim by means of experiments, showing that a significant improvement of users with disabilities results in the game play. We also observed that users with moderate cognitive impairment had bigger differences between feedback conditions than users with mild cognitive impairment (see Tables 1 and 2).

Different articles reviewed the importance of feedback in motor learning and rehabilitation. To our knowledge, there 
did not exist any study about the importance of the mirror feedback in vision-based rehabilitation systems. In fact, on one hand, [38] indicated that feedback might enhance motor leaning but there were many areas as yet not examined as the case of mirror feedback. On the other hand, [39] reviewed different types of natural visual feedback such as superposition, side-by-side 3D perspective, end-effector movements, and third- and first-person perspective. However, they did not reference any work about mirrors as natural visual feedback. For this reason, with the aim of seeking deeply articles related to mirror feedback, we also searched at Google Scholar, Web of Science, IEEE Explorer, and ACM Digital Library different combinations of the following key words: feedback, motor learning, augmented feedback, extrinsic feedback, and rehabilitation and mirror. We did not find any article related to mirrors as natural visual feedback, either.

Finally, we want to discuss the relationship between the mirror feedback and the game feel definition used for game design [49]. Game feel is the sensation of the system's response to the player: the kinesthesic qualities of the experience created by coupling with the input device and seeing what happens in the game as a result. In our experience, the input device is the user's own body; therefore, it makes sense that feedback should be related to it as well. In this sense, it is interesting to point out that the results obtained for the control group users (users without disabilities) could be interpreted to mean the mirror feedback mechanism is not significant. However, it should be taken into account that the video game was specifically designed for users with disabilities and its game play was too easy for users without disabilities. It may be interesting to perform another user study with more complex games based on vision-based interaction, in order to properly explore if the introduction of mirror feedback can improve the user experience of vision-based interaction.

Another limitation of our study was the sample size of users with disabilities ( 8 subjects). However, a post hoc power analysis indicated that with 8 subjects there would be a $90 \%$ chance (for $\alpha=0.05$ ) that the statistics would have detected a difference greater than 6.6 points in the time-to-start measure (we obtained a mean difference of 12.5) and greater than 50 points in the time-to-complete measure (we obtained a mean difference of 50.38).

\section{Conclusion}

In this work, we described a feedback implementation that improved results in rehabilitation sessions for users with disabilities, for this reason we recommend developers and researchers use this improvement in vision-based motor rehabilitation interactive systems. We experimented mirror feedback by means of a user study; we considered that it was an appropriate scenario to explore mirror feedback in video games. This claim was supported by the fact that the user's psychological conditions, with mild to moderate cognitive impairment, could mean that the normal feedback of current games was not enough for understanding the game play. In this case, therapy objectives lost effectiveness.

This was particularly interesting in rehabilitation games because the advantage of observation and imitation for learning was known and also because mirror movements and imitation learning was recommended in motor rehabilitation. For this reason, mirrors equip motor therapy rooms and they allow the patients to see themselves in order to perform correctly the therapy.

We observed that users with cognitive impairment had bigger differences between feedback conditions. The higher cognitive impairment user had, the more important feedback was in order to perform correctly the therapy. Results confirmed our hypothesis that in case of disabilities the mirror feedback mechanisms facilitated the interaction in the visionbased systems for rehabilitation. Once the positive effects of mirror feedback mechanism in rehabilitation system were demonstrated, it may potentially be extended to individuals with disabilities, in order to help achieving better functional abilities.

\section{Conflict of Interests}

The authors declare that there is no conflict of interests regarding the publication of this paper.

\section{Acknowledgments}

The authors thank the Spanish Association of Cerebral Palsy Centre (ASPACE) in the Balearic Islands for all of their collaboration, work, and feedback provided. This work was partially supported by the Projects 28/2011 (Ajudes grup competitiu UGIVIA) granted by the Govern de les Illes Balears, 19 MAEC-AECID A2/037538/11, and TIN2012-35427 of the Spanish Government, with FEDER support. The authors also thank the Mathematics and Computer Science Department at the University of the Balearic Islands for its support.

\section{References}

[1] K. Sung, "Recent videogame console technologies," Computer, vol. 44, no. 2, pp. 91-93, 2011.

[2] K. Tanaka, J. R. Parker, G. Baradoy, D. Sheehan, J. R. Holash, and L. Katz, "A comparison of exergaming inter- faces for use in rehabilitation programs and research," Loading, vol. 6, no. 9, 2012.

[3] R. A. Clark, Y.-H. Pua, K. Fortin et al., "Validity of the Microsoft Kinect for assessment of postural control," Gait and Posture, vol. 36, no. 3, pp. 372-377, 2012.

[4] B. Bonnechère, B. Jansen, P. Salvia et al., "Validity and reliability of the Kinect within functional assessment activities: comparison with standard stereophotogrammetry," Gait \& Posture, vol. 39, no. 1, pp. 593-598, 2014.

[5] R. A. Clark, Y. H. Pua, A. L. Bryant, and M. A. Hunt, "Validity of the Microsoft Kinect for providing lateral trunk lean feedback during gait retraining," Gait and Posture, vol. 38, no. 4, pp. 10641066, 2013.

[6] J. M. Cogollor, C. Hughes, M. Ferre et al., "Handmade task tracking applied to cognitive rehabilitation," Sensors, vol. 12, no. 10, pp. 14214-14231, 2012.

[7] S. Obdrzalek, G. Kurillo, F. Ofli et al., "Accuracy and robustness of kinect pose estimation in the context of coaching of elderly population," in Proceedings of the 34th Annual International Conference of the IEEE Engineering in Medicine and Biology Society (EMBS '12), pp. 1188-1193, IEEE, September 2012. 
[8] P. Rego, P. M. Moreira, and L. P. Reis, "Serious games for rehabilitation: a survey and a classification towards a taxonomy," in Proceedings of the 5th Iberian Conference on Information Systems and Technologies (CISTI '10), pp. 1-6, IEEE, June 2010.

[9] M. Sandlund, S. McDonough, and C. Häger-Ross, "Interactive computer play in rehabilitation of children with sensorimotor disorders: a systematic review," Developmental Medicine and Child Neurology, vol. 51, no. 3, pp. 173-179, 2009.

[10] B. Moyà-Alcover, A. Jaume-I-Capó, J. Varona, P. MartinezBueso, and A. Mesejo Chiong, "Use of serious games for motivational balance rehabilitation of cerebral palsy patients," in Proceedings of the 13th International ACM SIGACCESS Conference on Computers and Accessibility (ASSETS '11), pp. 297-298, New York, NY, USA, October 2011.

[11] N. Maclean, P. Pound, C. Wolfe, and A. Rudd, "The concept of patient motivation: a qualitative of stroke professionals' attitudes," Stroke, vol. 33, no. 2, pp. 444-448, 2002.

[12] F. Andersona, M. Annetta, and W. F. Bischofa, "Lean on wii: physical rehabilitation with virtual reality and wii peripherals," Annual Review of CyberTherapy and Telemedicine, vol. 8, no. 1, pp. 181-184, 2010.

[13] M. Sandlund, E. L. Waterworth, and C. Häger, "Using motion interactive games to promote physical activity and enhance motor performance in children with cerebral palsy," Developmental Neurorehabilitation, vol. 14, no. 1, pp. 15-21, 2011.

[14] A. L. Betker, A. Desai, C. Nett, N. Kapadia, and T. Szturm, "Game-based exercises for dynamic short-sitting balance rehabilitation of people with chronic spinal cord and traumatic brain injuries," Physical Therapy, vol. 87, no. 10, pp. 1389-1398, 2007.

[15] J. W. Burke, M. D. J. McNeill, D. K. Charles, P. J. Morrow, J. H. Crosbie, and S. M. McDonough, "Optimising engagement for stroke rehabilitation using serious games," Visual Computer, vol. 25, no. 12, pp. 1085-1099, 2009.

[16] S. Flynn, P. Palma, and A. Bender, "Feasibility of using the sony playstation 2 gaming platform for an individual poststroke: a case report," Journal of Neurologic Physical Therapy, vol. 31, no. 4, pp. 180-189, 2007.

[17] D. Goude, S. Björk, and M. Rydmark, "Game design in virtual reality systems for stroke rehabilitation," Studies in Health Technology and Informatics, vol. 125, no. 2007, pp. 146-148, 2007.

[18] D. Rand, R. Kizony, and P. L. Weiss, "Virtual reality rehabilitation for all: vivid gx versus sony playstation ii eyetoy," in Proceedings of the 5th International Conference on Disability, Virtual Environments and Associated Technologies, pp. 87-94, Citeseer, 2004.

[19] A. Jaume-i-Capó, P. Martinez-Bueso, B. Moya-Alcover, and J. Varona, "Interactive rehabilitation system for improvement of balance therapies in people with cerebral palsy," IEEE Transactions on Neural Systems and Rehabilitation Engineering, vol. 22, no. 2, pp. 419-427, 2014.

[20] J. E. Deutsch, M. Borbely, J. Filler, K. Huhn, and P. GuarreraBowlby, "Use of a low-cost, commercially available gaming console (Wii) for rehabilitation of an adolescent with cerebral palsy," Physical Therapy, vol. 88, no. 10, pp. 1196-1207, 2008.

[21] A. Da Gama, T. Chaves, L. Figueiredo, and V. Teichrieb, "Guidance and movement correction based on therapeutics movements for motor rehabilitation support systems," in Proceedings of the 14th Symposium on Virtual and Augmented Reality (SVR '12), pp. 191-200, Rio Janiero, Brazil, May 2012.

[22] B. Lange, S. Koenig, E. McConnell et al., "Interactive gamebased rehabilitation using the microsoft kinect," in Proceedings of the 19th IEEE Virtual Reality Conference (VR '12), pp. 171-172, Washington, DC, USA, March 2012.

[23] Y.-J. Chang, S.-F. Chen, and J.-D. Huang, "A Kinect-based system for physical rehabilitation: a pilot study for young adults with motor disabilities," Research in Developmental Disabilities, vol. 32, no. 6, pp. 2566-2570, 2011.

[24] L. Pareto, J. Broeren, D. Goude, and M. Rydmark, "Virtual reality, haptics and post-stroke rehabilitation in practical therapy," Proceedings of the ICDVRAT with ArtAbilitation Conference, 2008.

[25] K. Laver, S. George, S. Thomas, J. E. Deutsch, and M. Crotty, "Virtual reality for stroke rehabilitation," Stroke, vol. 43, no. 2, pp. e20-e21, 2012.

[26] A. Matthew, "A scoping review of exergaming for adults with systemic disabling conditions," Journal of Bioengineering \& Biomedical Science, 2011.

[27] M. Turk, "Computer vision in the interface," Communications of the ACM, vol. 47, no. 1, pp. 60-67, 2004.

[28] Y. Jung, S.-C. Yeh, and J. Stewart, "Tailoring virtual reality technology for stroke rehabilitation: a human factors design," in Proceeding of the Extended Abstracts on Human Factors in Computing Systems (CHI EA '06), pp. 929-934, ACM, New York, NY, USA, April 2006.

[29] A. Jaume-i-Capó, B. Moyà-Alcover, J. Varona, P. MartinezBueso, and A. M. Chiong, "Motivational rehabilitation using vision-based serious games," Biomedical Engineering, vol. 764, no. 1, pp. 62-70, 2013.

[30] A. Reyes-Amaro, Y. Fadraga-Gonzalez, O. L. Vera-Perez et al., "Video games for motivational balance rehabilitation," in Proceedings of the e-Health and Environment Conference in the Middle East, pp. 127-133, 2012.

[31] A. Reyes-Amaro, Y. Fadraga-González, O. L. Vera-Pérez et al., "Rehabilitation of patients with motor disabilities using computer vision based techniques," Journal of Accessibility and Design for All, vol. 2, no. 1, pp. 62-70, 2013.

[32] G. Wulf, S. Chiviacowsky, and R. Lewthwaite, "Normative feedback effects on learning a timing task," Research Quarterly for Exercise and Sport, vol. 81, no. 4, pp. 425-431, 2010.

[33] M. Iacoboni and J. C. Mazziotta, "Mirror neuron system: basic findings and clinical applications," Annals of Neurology, vol. 62, no. 3, pp. 213-218, 2007.

[34] D. Broetz and N. Birbaumer, "Behavioral physiotherapy in post stroke rehabilitation," NeuroRehabilitation, vol. 33, no. 3, pp. 377-384, 2013.

[35] S. Cho, J. Ku, Y. K. Cho et al., "Development of virtual reality proprioceptive rehabilitation system for stroke patients," Computer Methods and Programs in Biomedicine, vol. 113, no. 1, pp. 258-265, 2014.

[36] K. Eng, E. Siekierka, P. Pyk et al., "Interactive visuo-motor therapy system for stroke rehabilitation," Medical \& Biological Engineering \& Computing, vol. 45, no. 9, pp. 901-907, 2007.

[37] E. B. Nash, G. W. Edwards, J. A. Thompson, and W. Barfield, "A review of presence and performance in virtual environments," International Journal of Human-Computer Interaction, vol. 12, no. 1, pp. 1-41, 2000.

[38] P. M. van Vliet and G. Wulf, "Extrinsic feedback for motor learning after stroke: what is the evidence?" Disability and Rehabilitation, vol. 28, no. 13-14, pp. 831-840, 2006.

[39] R. Sigrist, G. Rauter, R. Riener, and P. Wolf, "Augmented visual, auditory, haptic, and multimodal feedback in motor learning: a review," Psychonomic Bulletin and Review, vol. 20, no. 1, pp. 21-53, 2013. 
[40] B. L. Chan, R. Witt, A. P. Charrow et al., "Mirror therapy for phantom limb pain," The New England Journal of Medicine, vol. 357, no. 21, pp. 2206-2207, 2007.

[41] K. W. Krigger, "Cerebral palsy: an overview," The American Family Physician, vol. 73, no. 1, pp. 91-100, 2006.

[42] J. Charles and A. M. Gordon, "Development of hand-arm bimanual intensive training (HABIT) for improving bimanual coordination in children with hemiplegic cerebral palsy," Developmental Medicine and Child Neurology, vol. 48, no. 11, pp. 931936, 2006.

[43] A. M. Gordon, Y. C. Hung, M. Brandao et al., "Bimanual coordination in children with hemiplegic cerebral palsy. A randomized trial," Neurorehabilitation and Neural Repair, vol. 25, no. 8, pp. 692-702, 2011.

[44] A. Jaume-i-Capó, B. Moya-Alcover, and J. Varona, "Design is sues for vision-based motor-rehabilitation serious games," in Technologies of Inclusive Well-Being, pp. 13-24, Springer, New York, NY, USA, 2014.

[45] L. A. King and F. B. Horak, "Delaying mobility disability in people with parkinson disease using a sensorimotor agility exercise program," Physical Therapy, vol. 89, no. 4, pp. 384-393, 2009.

[46] T. N. Tombaugh and N. J. McIntyre, "The mini-men tal state exam ination: a comprehensive review," Journal of the American Geriatrics Society, vol. 40, no. 9, pp. 922-935, 1992.

[47] J. Varona, A. Jaume-i-Capó, J. Gonzàlez, and F. J. Perales, "Toward natural interaction through visual recognition of body gestures in real-time," Interacting with Computers, vol. 21, no. 1-2, pp. 3-10, 2009.

[48] N. Bevan, "Measuring usability as quality of use," Software Quality Journal, vol. 4, no. 2, pp. 115-130, 1995.

[49] S. Swink, Game Feel: A Game Designer's Guide to Virtual Sensation, Morgan Kaufmann, 2008. 

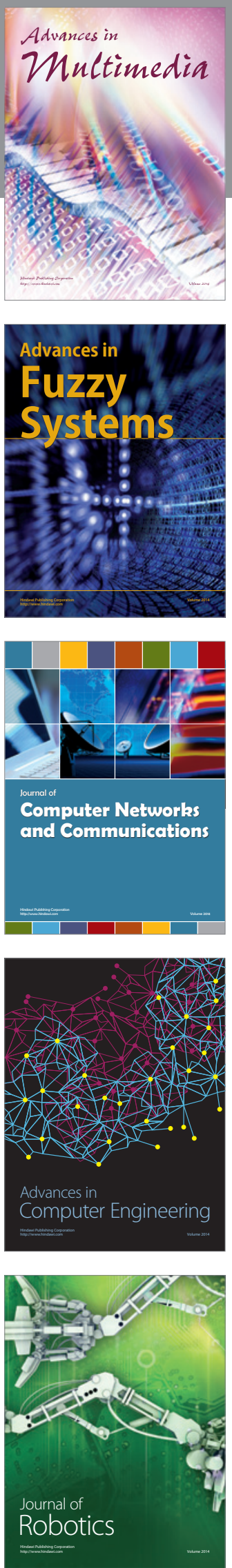

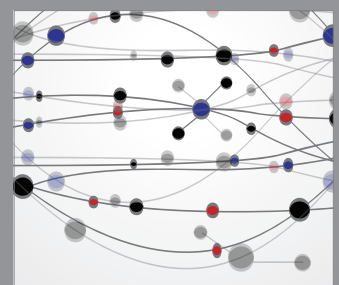

The Scientific World Journal
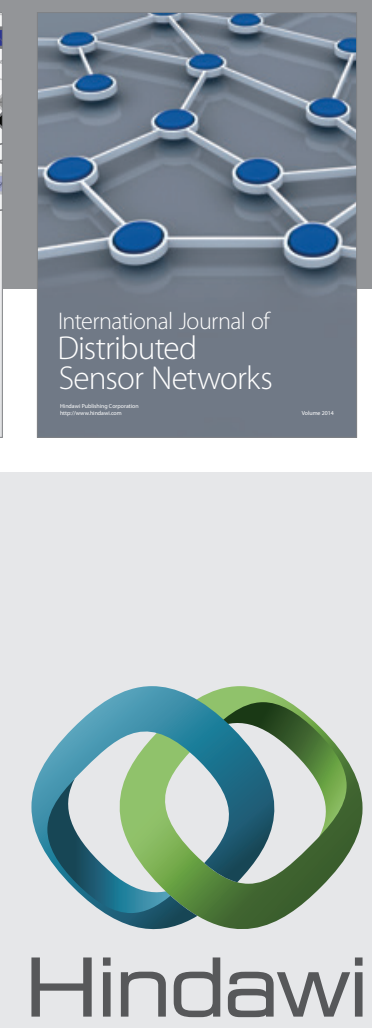

Submit your manuscripts at

http://www.hindawi.com
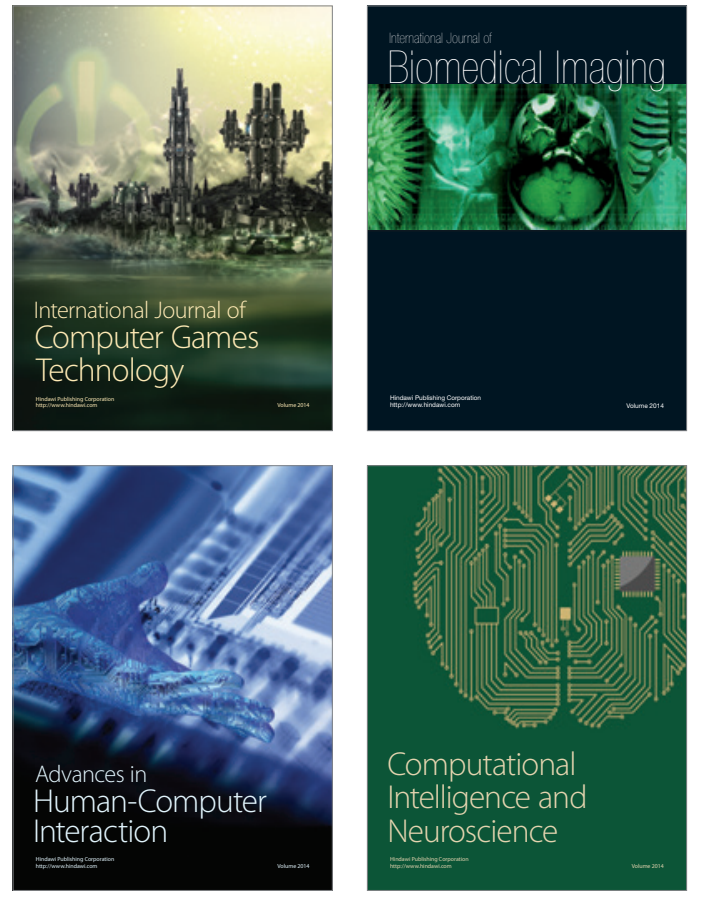
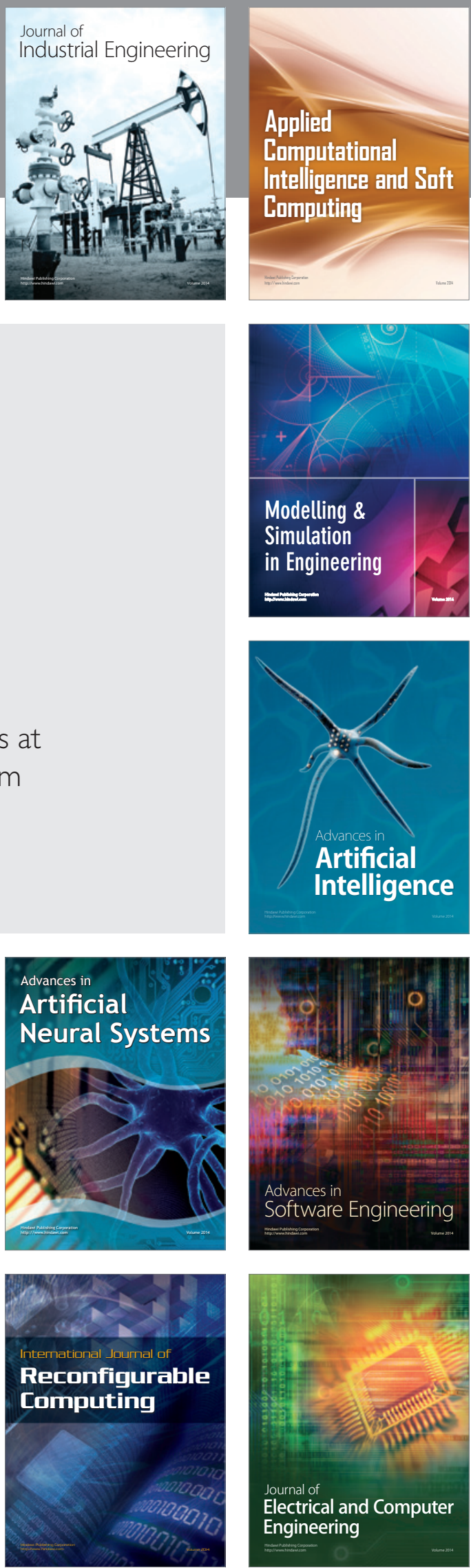